\title{
Evaluation of Midwives' and Nurses' Continuing Professional Development in Reducing Maternal and Neonatal Mortality in Embu County, Kenya
}

\author{
Lucy K. Gitonga, Njogu Samson Muriuki \\ Chuka University, Chuka, Kenya \\ Email: gitonga30@yahoo.com \\ Received 18 January 2014; revised 16 February 2014; accepted 24 February 2014 \\ Copyright (C) 2014 by authors and Scientific Research Publishing Inc. \\ This work is licensed under the Creative Commons Attribution International License (CC BY). \\ http://creativecommons.org/licenses/by/4.0/

(c) (i) Open Access

\begin{abstract}
Continuing professional development (CPD) is one of the principal means by which health professionals (Nurses and midwives) maintain, improve, and broaden the knowledge and skills required for optimal patient care and safety, to be more specific reduction of maternal and neonatal mortality and morbidity. However, the lack of a widely accepted instrument to assess the impact of CPD activities on clinical practice thwarts researchers' assessment of the effectiveness of CPD activities. The objective of the study is to develop a theory and practice-based, valid, reliable national instrument to assess the impact of accredited CPD activities on reducing maternal and neonatal mortality in Kenya using an integrated model for the study of healthcare professionals' behavior through environmental scanning of the factors that promote good performance. The researcher will analyze the instruments identified in a systematic review of factors motivating and demotivating nurses and midwives' behaviours using criteria that reflect the literature on measurement development of CPD leaders and providers' priorities. The outcome of this phase will be an inventory of instruments using a competence-based model. Working from this inventory, the most relevant items for assessing the concepts listed will be selected. Then, the researcher will verify whether these items are acceptable or need modification, what aspects need revision, and whether important items are missing and should be added. The outcome of this phase will be a new national instrument integrating the most relevant tools to fit our integrated model of healthcare professionals' behavior. Two data collections are planned: 1) pretesting of the new instrument, to assess its reliability and validity and 2) a study using the instrument before and after CPD activities with randomly selected groups, one acting as control group to assess measurement effect. The researcher will conduct individual interviews and focus groups with CPD providers and leaders to identify anticipated barriers and enablers for implementing the new instrument in CPD practice. Drawing on the results from the previous phases, we will use consensus-building methods with
\end{abstract}


the decision makers to develop a plan to implement the new instrument.

Keywords

Evaluation; Continuing Professional Development; Maternal Mortality; Neonatal Mortality

\section{Introduction}

\subsection{Background}

"Evaluation is as a basic to professional development as it is to education". Unfortunately, as is so often the case in nursing and midwifery, systematic evaluations of professional development programs are rarely undertaken. According to [1] millions of dollars have been provided in the name of professional development, but the quality of these programs goes virtually unchallenged.

The effectiveness of the nurse/midwife has traditionally been associated with maintaining standards of care. Continuing professional development (CPD) is essential to ensure this important group feels adequately prepared to perform their role and has been recognized as an important factor in maintaining job satisfaction and reducing wasteful staff turnover (Jill 2010). Additionally, a review of the literature indicates that the area of nurses' and midwives' continuing professional development (CPD) is of growing interest in Kenya and internationally. However, while an increasing range of literature focuses on particular aspects of CPD, there is a limited literature on the evaluation of the impact of these trainings on improvement of maternal and child health [1] [2]. Review of literature also reveals that although many countries are struggling to train, evidence shows that training does not march with competence. While most CPD experiences might be considered as means of introducing or enhancing knowledge, skills and attitudes, it cannot be assumed that this is achieved. Moreover many researchers have argued that it is not merely the type of professional knowledge being acquired that is important, but the context through which it is acquired and subsequently used that actually helps us to understand the effectiveness of that knowledge [3].

Analyzing the means through which CPD for nurses and midwives is organized and structured may help us to understand not only the motivation behind such structures, but also the nature of professional knowledge and professionalism acquired itself. Although participation in CPD is currently compulsory in Kenya defining the sanctions or implications for non participation remains to be defined and even if it is there it is in paper and not implemented. The healthcare landscape is diverse and complex. In Kenya there is currently no commonly accepted approach to lifelong learning. However, there is broad agreement that patients are best served when those who care for them maintain competence by engaging in continuous learning and assessment strategies [4]. Optimally, these strategies would be "highly self-directed" with content, learning methods, and learning resources selected specifically for the purpose of improving the knowledge, skills, and attitudes that nurses and midwives require in their daily professional lives that lead to improved patient outcomes.

According to [4]. CPD can be defined as a continuing process, outside formal undergraduate and postgraduate training, that enables individual health workers to maintain and improve standards of medical practice through the development of knowledge, skills, attitudes and behaviour, CPD should also support specific changes in practice. Arising from the literature review, it is considered that the definition of CPD could be divided into two parts: gaining knowledge and improving patient care.

Additionally, the procedures for recording CPD were perceived as needing to address both of these aspects. However, a body of literature claimed that a gain in knowledge does not necessarily result in a change in behaviour by the clinician [4] [5]. If that is so, then other questions arise: will an improvement in patient care occur? And, if it does, how can it be measured [6]? Moreover, literature review has revealed that there is little literature on the effectiveness of CPD in improving patient care [7]. However in his work, he stated that: effective CPD involves both "learning" and being "fit to practise". Knowing both the "why" and the "how", and putting learning into practice, the effectiveness is facilitated when professionals are able to determine their own learning needs through reflection within the totality of their practice. This means being able to go beyond what is quantifiable.

A responsive health system is one offering appropriate antenatal care; comprehensive emergency obstetrics 
care and quality delivery services. Maternal and neonatal morbidity and mortality levels continue to be recognized internationally as public health priorities. Moreover these indicators in Africa have continued to rise instead of declining since the launch of Safe Motherhood Initiative 15 years ago. Of all maternal deaths occurring globally, 99\% of them occur in developing countries with Sub-Saharan Africa having the highest maternal mortality ratio of 900 maternal deaths per 100,000 live births and also the highest life time risk of maternal death of 1:26 [8]. The idea that health professionals should be accountable to the society they serve is not a new concept and for a long time [6] the continuing professional development (CPD) of health professionals has been seen as one way in which population's level of health could be improved.

The public was, and is still today, increasingly demanding a system that is more responsive to regional and community needs. As a result, there is a need for more health professional education at all stages of the education continuum - undergraduate, postgraduate, and continuing professional development-which meets the health and social needs of the populations being served. The trend is now towards "socially accountable" health care, meaning that the broader context of CPD must also include the personal, social, and political aspects of health care and as such, involve a widening of accountability to patients, the community, managers and policymakers [9] CPD planning must take into account local and national priorities as well as personal learning needs. There is plethora of activity both globally and locally geared towards addressing maternal and neonatal morbidity and mortality. This has placed the role of midwives in sharp focus as governments think of ways of ameliorating this problem. Since the number of midwives in sub-Saharan Africa and Kenya in particular, are limited, innovative ways of improving their knowledge and skills in highly effective targeted obstetric and newborn interventions without removing them from the workplace are desirable [10]. Midwives offer evidence-based, cost-effective high impact care [11] [12]. Moreover as competent care providers, midwives detect problems early in complicated births, take appropriate life-saving actions immediately and refer where appropriate. Additionally, their critical thinking and decision making skills contribute to the saving of lives by making timely decisions and action taking [13].

In this regard therefore, there is need to strengthen their skills through continuing professional development(CPD).Anecdotal evidence suggest that increasing demands on service delivery have reduced time available for CPD activities in many countries coupled with less support from service providers in relation to staff needs [14]. Researches on CPD have been done in other countries but no documented evidence in Kenya to critically analyze the perspectives of CPD practice for Nurses and midwives and their impact in achievement of MDG 4 and 5. Moreover the fast generation of overwhelming information of health and complexity in technologies in the $21^{\text {st }}$ century poses the greatest challenge to health care workers ability to provide effective and efficient high quality and up to date health care services.

\subsection{Statement of the Problem}

The knowledge and skills acquired at the end of formal undergraduate and postgraduate professional medical education are insufficient to sustain competence and performance over a career. Either through participating in organized continuing education programs or through individual learning activities, healthcare professionals are expected to remain current in their practice through efficient knowledge management practices (evidence-informed practice) and self-directed learning strategies (lifelong learning). Healthcare professionals are expected to effectively engage in lifelong learning strategies in a rapidly changing healthcare system that is increasingly strained due to underfunding, inadequate health care workers and limited access to data on performance or current health outcomes. Further compounding these challenges is the increasing scrutiny of professional and public concerns related to the variability in the quality of care provided, the safety of the health system, and the frequency of adverse events. Collectively these concerns have fostered a growing emphasis on the need for accountability within the health professions for the continued privilege of self-regulation.

Despite the increasing relevance of CME and the implementation of revalidation Kenya, region specific data remains scarce [15]. What is well understood however is that CME regulations across the country vary providing a challenge to the harmonization of nurses and midwives' learning and practice across the country? Although CME will remain a national responsibility there are currently no standards governing the management by nursing council, there are no standard tools to guide evaluation of effectiveness of CPD in improving patients care particularly with regards to reducing maternal and neonatal care. The available tools focus mainly on the achievements the nurse and midwife has made by participating in a CPD activity rather than focusing on how 
effective the knowledge and skills gained will be used to impact on health care.

Despite recent developments, health care in Kenya remains suboptimal. In a country with a population a population of approximately 38 million [16], there are currently an estimated 54 nurses/100,000 population which is far below the World Health recommendation. Maternal mortalities in Kenya have remained increasing high since 1998 to 488 maternal deaths per 100,000 live births in the year 2008/2009 (with some regions reporting maternal mortalities of over 1000/100,000 live births). Neonatal mortality rate has recorded a marginal reduction from $33 \%$ in 2003 to 31\% in 2008/2009 compared to other indicators of child survival. Embu County is one of the counties where a lot of CPD has been done for nurses and midwives particularly those focusing on reducing maternal and neonatal deaths. However there is no research indicating whether this training has negatively or positively impacted on these deaths.

\subsection{Purpose}

The broad objective of the study will be to evaluate the impact of CPD activities for nurses and midwives in reducing maternal mortality in Embu County, Kenya by developing an evaluation tool.

\subsection{Objectives}

The specific objectives of this study will be to:

1) Analyze the current tools used to evaluate the impact of CPD practice in the county.

2) Developed tool for evaluating the effectiveness of CPD practice towards reducing maternal and child mortality in Kenya.

\subsection{Study Questions}

The study will be guided by the following research questions:

1) Are there any existing tools to develop the impact of CPD for nurses and midwives in Embu County?

\subsection{Study Hypothesis}

1) The measurement instrument will correlate in the expected direction with self-reported behaviour as well as with changes in self-reported behaviour.

\subsection{Significance}

The findings of the study are expected to inform the government and the community on the scope of evaluation of CPD practice by nurses and midwives in Kenya. As a result a national tool will be developed which will guide in the evaluation of CPD activities in reducing maternal and neonatal mortality in the country. Consequently this will have financial implication in that money will be directed in the right places because there will be clear indication of the expected results as evidence will have been obtained.

\subsection{Scope}

The study will be carried out in Embu County, Kenya. The study will involve nurses and midwives working in one maternity unit in the county. It will also involve policy makers and the regulatory body, the Nursing council of Kenya officers.

\subsection{Limitations and Delimitations}

Some staff that will be expected to be on duty that time might be off for any unavoidable circumstances. The nursing officer in charge will be informed to replace them by making sure that others come to fill in the vacant position in the duty roster.

\subsection{Assumptions}

1) The respondents would give accurate and honest information.

2) There is no national tool to evaluate the impact of CPD practice on reducing maternal and neonatal mortal- 
ity in Kenya.

\subsection{Definition of Terms}

The following terms are defined in the study as:

Essential maternal and newborn care Skills-skills required in provision of prenatal, natal and postnatal care of both mother and baby.

Midwife-A person qualified to practice midwifery, having received specialized training in obstetrics and child care.

Evaluation-is a systematic determination of a subject's merit, worth and significance, using criteria governed by a set of standards.

Impact-Measure of the tangible and intangible effects (consequences) of one things or entity's action or influence upon another.

Maternal mortality-The number of registered maternal deaths due to birth- or pregnancy-related complications.

Neonatal mortality—death of a live born infant within the first 28 days of life death.

\section{Literature Review}

\subsection{Introduction}

Showing the impact of learning is important as it allows the customer to know exactly how the learning process will bring positive results to the business. However, while the business units value the business linkage (impact or outcome) and evaluation (measurement) the most, learning departments often spend the least amount of time and resources on these two activities.

In Kenya, it is assumed that continuing professional development (CPD), which encompasses continuing medical education (CME) [14] plays an important role in maintaining and improving the quality and efficiency of the healthcare system [22] by translating evidence into clinical practice [17]-[20]. In other words, CPD serves as an important knowledge-translation strategy and is one potential approach that could be incorporated into the Knowledge to Action Process (KTA) framework [20]. The KTA framework, which explains how knowledge is produced and implemented in healthcare, contains two parts: the knowledge creation cycle and the action cycle [21]. While the first cycle comprises the process of creating knowledge, the second one constitutes the process of applying the knowledge thus created. By translating knowledge and evidence into practice, CPD pertains to the action cycle [22]. Designed to improve performance in healthcare practices and, ultimately, health outcomes, CPD strategies follow the dynamic and iterative process for knowledge translation.

As in other educational disciplines, most evaluation frameworks used in CPD are derived from Kirkpatrick's model [23]. This model assesses training effectiveness by measuring participants' reactions to an educational activity (level 1); changes in participants' knowledge, skills, or attitudes (level 2); transfer of learning to practice/ observed changes in behaviour (level 3); and finally, the results of the newly acquired behaviour on organizational outcomes such as productivity and quality (level 4). According to this model, the effects of current approaches to the assessment of the impact of accredited.

CPD activities should ideally be evaluated focusing on participants' participation, satisfaction, and changes in knowledge, behaviour, and patient outcomes [24]. In practice, however, most CPD providers only assess levels 1 and 2 outcomes using pre- and post activity self-administered questionnaires. Although the impacts of levels 3 and 4 have been measured in the context of research projects using health services methods [25]. CPD providers are still struggling to find reliable ways to measure these impacts on a routine basis.

[26] Proposed an integrated conceptual model to predict behaviour change in healthcare professionals that offers a clear basis for developing a valid and reliable measurement instrument to assess CPD impacts on clinical practice (Kirkpatrick's level 3 outcomes).

Since individual decisions are often central to the adoption of clinically related behaviours, theories providing information about cognitive mechanisms underlying behaviours help provide direction to behaviour-change interventions targeting healthcare professionals. According to Training and Development Agency for schools [26], to evaluate the impact of professional development it is crucial to consider what was intended to be achieved, and what impact could reasonably be expected, in any given time frame. This agency stated that for CPD pro- 
gram to be effective certain principles have to be followed:

1) Planning for CPD and the evaluation of its impact should be integral to performance management.

2) Impact evaluation should focus on what participants learn, how they use what they have learned, and the effect on the learning of children and young people.

3) There should be an agreed timeline for evaluating outcomes, accepting that some outcomes, such as children and young people's improved performance, may take longer to become evident than others. Unanticipated outcomes will also be considered by the review.

4) Planning and implementation of the impact evaluation should be a collaborative process between the individual and key staff involved in performance management and/or coaching and mentoring.

5) The evidence base and the success criteria for the evaluation of impact should be agreed.

6) Impact evaluation should be considered in the short, medium and long term. Longer-term professional development activities should involve formative reviews of impact at agreed stages.

7) The evaluation of impact should include a cost-benefit analysis of the professional development.

8) The processes for evaluating the impact of CPD activities need to be reviewed regularly to ensure that they are effective and proportionate.

\subsection{Evaluating CPD: Enablers and Challenges}

It is clear that there are a wide variety of levels at which CPD can be evaluated. It is also clear that most useful evaluations combine methods, marrying the rigor of quantitative measures to the deeper formative information provided by qualitative methods, a process sometimes known as "holistic" evaluation .Especially where CPD programs are complex and multifaceted, this needs to be reflected in evaluation strategies, with methods appropriate for each component. Evaluation of CPD will usually want to serve two main purposes: summative evaluation (does the program improve outcomes?) and formative assessment (how can the program are improved?). These two goals can best be served by collecting data in different ways, test scores for example often being used summatively while interview and survey data can be used to guide formative evaluation. A further point is that in order to minimize bias, data needs to be collected from a variety of stakeholders, rather than just one group, and to use a variety of research method [26].

Evaluation can be carried out either entirely in-house or with the help of external experts. When pure in-house evaluation is carried out, evaluation capacity must exist, and where necessary be developed through professional development [27]. When external evaluation is preferred, it is important to ensure that participants contribute to evaluation design and activities, as use of evaluation results has often been found to be patchy where that is not the case [27], Furthermore, it has been found that where participants themselves are not involved in developing evaluation, they are less likely to take account of evaluation information to change their practice.

As well as providing continuous feedback, evaluation anxiety can be reduced by stressing positive as well as negative outcomes, involving stakeholders in evaluation, clearly explaining the purpose of the evaluation and discussing the purpose of the evaluation and prior experiences of evaluation with stakeholders [28].

Evaluation at best will provide not just an overview of whether CPD itself has been successful, but will also have strong positive learning benefits to teachers in the school [28]. To be most effective evaluation processes need to be embedded in the school and just not added on at the end of a CPD programme of activity.

\subsection{Limitations of Evaluating CPD}

While the ultimate purpose of CPD is to secure changes in classroom practice that will have a positive impact, directly or indirectly, on student learning the evaluative evidence to support this relationship appears to be less forthcoming. The current CPD evaluation processes would appear to be restricted in a number of ways. Firstly, it is clear that most evaluation models applied to CPD overlook or omit the issue of cost effectiveness [28]. Point out that CPD should not be undertaken if the costs to the system outweigh the benefits. Yet in evaluating the impact of CPD in schools the issue of cost effectiveness is rarely explored. As a result, we know relatively little about the cost effectiveness of alternative forms of CPD. Secondly; CPD evaluation processes are rarely fine grained or sufficiently robust enough to capture evidence about the relationship between CPD and learning outcomes. It would appear that there are major limitations in the evaluation methodologies employed in schools. [28] has suggested that there are three major weaknesses of the evaluation processes applied to CPD, also found in [28]. 


\subsection{Continuing Professional Development Practice}

The area of nurses' and midwives' continuing professional development (CPD) is of growing interest in Kenya and internationally. However, while an increasing range of literature focuses on particular aspects of CPD, there is a limited literature on the follow up of the implementation of the laid down policies particularly those addressing MDGs 5. This article examines the current practice of CPD by nurses and midwives and will propose a framework through which follow up can be done. CPD can be structured and organized in a number of different ways, and for a number of different reasons. While most CPD experiences might be considered as means of introducing or enhancing knowledge, skills and attitudes, it cannot be assumed that this is uncontested. For example, [28] argues that it is not merely the type of professional knowledge being acquired that is important, but the context through which it is acquired and subsequently used that actually helps us to understand the nature of that knowledge. Analyzing the means through which CPD for nurses and midwives is organized and structured may help us to understand not only the motivation behind such structures, but also the nature of professional knowledge and professionalism itself [28]. Identifies three major contexts in which professional knowledge is acquired-the academic context, institutional discussion of policy and practice and practice itself).

Clearly, knowledge acquisition is not situated exclusively within anyone of these three contexts, but the identification of the different contexts is useful in analytical terms. Additionally, various strategies can be used to enable nurses undertake Continuous Professional Development [28]. State that Continuous Professional Development can be formal or informal whereby learning can take place in formal setting such as module courses at diploma, degree and higher degree levels, study days and half-day shorter programmes. A study done in Malawi [28] to describe the current status of CPD of health care personnel within MOH revealed that health workers practiced their CPD mainly through reading professional journals and attending seminars and workshops although all of them expressed the need to receive these journals for free and very few were willing to pay. Additionally, a study done to identify training and development needs of midwives in Indonesia by [29] revealed that although nurses were practicing CPD, all of the midwives expressed the need for further development and training with junior midwives expressing more needs. In Kenya, nurses are encouraged to upgrade themselves through distance learning \& part-time learning so as to address the shortage of staff, which is a key to quality of care in the health care systems although there is no clear monitoring to show whether this development is needs driven or not. This means that for one to undergo Continuous Professional Development one does not necessarily have to go to class [29]. For distance learning, one has to be away from his or her workstation during face-to face tutorials, study days or during exam times. For part time mode of study the off duty duration is shorter. On the other hand professional development by nurses can be hindered by poor support by their employers and these employers not making full use of their potential [29]. Found out that those employers must use this excellent opportunity of knowledge gained through professional development which will allow flexibility in employing nurses across shared work places. [29] suggested the following CPD activities which can help health professionals to update their knowledge and skills: self directed study, organizing study days, formal study, conference attendance, clinical supervision, and clinical activities, reading nursing articles/journals, and clinical activities. These will address the barriers that hinder nurses and midwives from participating in CPD. This is supported by [29] in a study on integrating performance and the practice of CPD among Canadian pharmacist who found out that pharmacist must practice informal CPD activities in order to continuously update and assure the public of the continuing quality of care provided by the practitioners.

Critical thinking and clinical actions empower the professional nurses and Continuous Professional Development may be the key to promoting and maintaining competency, strengthening the profession and improving quality of care [29] Mullins also suggested the following strategies: partner with staff to promote professional accountability through periodic self-evaluation, invite staff to share what they have learnt at continuing education activities, encourage staff to choose continuing education opportunities of interest which will promote attendance and professional accountability, make educational opportunities available at more than one time and allow participants to choose which time to attend and constantly seek and praise good work. In Kenya continuing professional development activities should be needs driven and a needs assessment should be done before coming up with the program. Additionally, selection criteria for nurses doing specialized courses have been set so that that who are interested in the area and are willing to share knowledge qualifies. More so the Ministry Of Health [29] Kenya has developed the following strategies in order to ensure that all staff participate in Continuous Medical Educations: post basic scholarship (though mainly for doctors), scholarships for short courses, 
specialized workshops, Continuous Medical Education at stations, conferences, on -job training budgetary provision made at departmental levels and giving its staff paid study leaves. To emphasize on this, the Ministry Of Health's (Kenya) future plans, include: Continuous Medical Education should be the basis for promotion and create more Continuous Medical Education opportunities. Additionally the managers should identify role development strategies for nurse manager educators and nurses who will offer employers, nurse managers, educators and nurses a starting point from which specific occupational competences can be further explored [29]. Moreover, there is need to develop more flexible courses targeted to actual rather than idealized [29] learning needs of each nurse and to allow more flexibility in their practice. This will be easy to use and can be designed to reflect what goals of practice are required by employers who are considering funding staff for professional development activities. Furthermore, nurses have curiosity and readiness to learn and know more about anything relating to their work. Therefore, being involved in any activities that would improve knowledge is an important component. These opportunities are used to compensate for shortcomings they had experienced in their initial training and or in-service programs mainly caused by lack of appropriate planning which is common in developing countries [29].

\section{Methodology}

\subsection{Research Design}

This will be a descriptive study utilizing interview checklist, questionnaire and focus group discussion since little is known about the study area in Kenya, that is, evaluation of the impact of CPD practice by nurses and midwives in Kenya (Wood and Ross 2006). This kind of a study design was chosen because the main aim will be to describe the phenomena in as much details as possible in terms of how they are and also explore the its perspectives in order to develop an evaluation tool. The environmental scan performed in this study will help determine the existing knowledge base regarding effective CPD for translating CPD for nurses and midwives into improved maternal and neonatal health. It will make it possible to identify the individuals or groups initiating CPD activities, the content and quality of CPD training, the strategies and means of conducting CPD training, and the impact of CPD training on fostering improved maternal and neonatal health care. Themes will also be described as they emerge. It will be phased.

\subsection{Location}

The study will be conducted in Embu Provincial General Hospital in Embu county, Kenya.

\subsection{Study Population and Selection Criteria}

Participants will be selected from nurses and mid-wives who have attended CPD in the past six months. A total of 200 nurses and midwives working in Embu Provincial General Hospital have attended CPD activities in the last six months.

\subsection{Sampling Procedures and Sample Size}

Sampling is the process of selecting few cases in order to provide information that can be used to make judgment about a much larger number of cases. The sampling in this study will be a random sampling of the nurses and midwives who have attended CPD activities. 132 respondents is a representative sample of a population of 200 members. Therefore, the research will randomly sample 140 respondents so as to cater for attrition and poorly responded questionnaires.

\subsection{Research Instruments}

The researcher will prepare a checklist to assess the existing evaluation tools. The existing tools will be retrieved for particulars about the intended tool to be developed. Several methods will be used to ensure the completeness of the information regarding each tool. If the tool does not provide detailed information, the authors will be contacted for clarification on the tool. This initial inventory will be enriched by updating the literature search and contacting experts in the field (CPD providers, health services managers, and the Nursing Council of Kenya) through phone calls and emails. The tools thus identified will be analyzed based on criteria that CPD decision 
makers deem important. Some of the criterion to be used will be the ability of the tool to be used by a large number of nurses and midwives in different clinical areas and contexts and also the capability of the tool being inexpensive to administer. To fulfill these tasks, two research assistants will independently extract data using a standardized form that will be revised at the beginning of the research project by team members to ensure that it fits decision makers' needs.

Once the information on the existing tools has been gathered, the researcher will then develop a new tool to collect the required information. The researcher will form a three-person Development Committee comprised of research assistants and CPD decision makers. They will examine each tool selected in phase 1 and choose the most relevant items to include in the new evaluation tool. Once this task is complete and all items will be integrated into a new national tool.

\subsection{Validity of the Instrument}

Validity is the ability of the instrument to test what it was intended to test. After preparing the research instruments, e-Delphi process will be used to check its face validity and likely acceptability and utility in CPD settings. The new instrument will also be examined for construct and content validity by experts from the Department of Nursing of The University of Nairobi.

Once the experts have provided their feedback, the Development Committee will review and make the final list of selected items grouped around variables of the integrated conceptual model.

\subsection{Reliability of the Instrument}

Reliability is ability of the instrument to collect data and give consistent results over and over again. To ensure reliability, the research will pilot the instrument in Chuka Level five hospitals. The hospital will be selected because the nurses and mid-wives share similar characteristics with those in Embu Provincial General Hospital. Data will be collected and split into two sets and analyzed. If the new evaluation tool gives reliability co-efficient of 0.7 and above, it will be considered reliable because according to reviewed literature an instrument with a reliability of 0.7 is reliable.

\subsection{Data Collection Procedures}

The research will follow all the procedures put in place by the University of Nairobi before going out into the field to collect data. Once permission has been granted, the researchers will then visit Embu Provincial General Hospital and seek permission to collect data. The researcher will randomly sample the participant for the study. The sampled participants will be asked to a complete a consent form before participating in the study. Once they have signed the consent form, they will then be given the evaluation tool developed to complete.

\subsection{Data Analyses}

Data collected will be cleaned and coded. Using Statistical Package for Social Sciences (SPSS), Data collected will then be analyzed using descriptive and inferential Statistics. Data will then be presented to the stakeholders for adoption of the evaluation tool.

\subsection{Ethical Consideration}

Ethics approval will be sought from The Ethics and Research Committee, Kenyatta National Hospital/University of Nairobi and Ministry of Health. All participants will be informed about the study and participation will be on voluntary basis. Written consent from the participants will be obtained and participants will be free to withdraw from the study at any point. Data obtained will be stored safely for the specific period of time according to the requirements of the ethics committee. The filled questionnaires will be sealed by the respondents and handed over to be opened by the researcher only. The data obtained will be anonymous since no writing of names. All the contact letters will be written on official headed paper and signed in blue ink by the researcher.

\section{References}

[1] Aileen, K. (2005) Models of Continuing Professional Development: A Framework for Analysis. Journal of In-Service 
Education, 31, 235.

[2] Danielson, E. and Hallin, K. (2008) Registered Nurses’ Perceptions of Their Work and Professional Development. Journal of Advanced Nursing, 61, 62-70. http://dx.doi.org/10.1111/j.1365-2648.2007.04466.x

[3] Fleet, J., et al. (2008) Continuing Professional Development and Social Accountability: A Review of the Literature. BMA Informa Health, 22, 15-19.

[4] Galer, J.B., et al. (2005) Managers Who Led. A Handbook for Improving Health Services. Cambridge.

[5] Gitonga, L.K. (2008) Positive Work Environment. Kenya Nursing Journal, 37, 13-15.

[6] Godin, G., Belanger-Gravel, A., Eccles, M. and Grimshaw, J. (2008) Healthcare Professionals' Intentions and Behaviours: A Systematic Review of Studies Based on Social Cognitive Theories. Implement Science, 3, 36. http://dx.doi.org/10.1186/1748-5908-3-36

[7] Hallin, K. and Danielson, E. (2007) Registered Nurses Perception of Their Work and Professional Development. Journal of Advanced Nursing. Online Early Articles. Published Article Online 22 Nov 2007.

[8] Hennessy, et al. (2006) The Training and Development Needs of Midwives in Indonesia: Paper 1 of 3. Human Resource Health, 4, 8. http://dx.doi.org/10.1186/1478-4491-4-8

[9] Jill, S., et al. (2010) The Effectiveness of Continuing Professional Development. A Report Prepared on Behalf of College of Emergency Medicine, Federation of Royal Colleges of Physicians and Manchester Metropolitan University.

[10] Kaluzny, D. (2006) Health Care Management. Organization Design and Behavior. 5th Edition, Library of Congress.

[11] Kenya Demographic Health Survey 2008-2009 (2010) 455 p.

[12] Khomeiran, H. (2006) Practice Development and Health Care Governance; a Recipe for Modernization. Journal of Nursing Management, 12, 137-146.

[13] Kirwan, A. and Adams, J. (2008) Nurse Education Today. Students’ Views of Enquiry-Based Learning in a Continuing Professional Development Module.

[14] Légaré, et al. (2011) Implementation Science 2011, 6:17 http://www.implementationscience.com/content/6/1/17 http://dx.doi.org/10.1186/1748-5908-6-17

[15] Legreid, A., et al. (2010) A Five-State CPD Pilot Program for Practising Pharmacists. The American Journal of Pharmaceutical Education, 74, 28. http://dx.doi.org/10.5688/aj740228

[16] Munro, M. (2008) Nursing Education Today Continuing Professional Development and the Charity Paradigm: Interrelated Individual, Collection and Organization Issues about Continuing Professional Development.

[17] Ministry of Health (2007) National Reproductive Health Policy. Enhancing Reproductive Health Status for all Kenyans.

[18] Ministry of Health (2006) National Nursing Standards and Guidelines Support Supervision Manual for Nurse Supervisors in Kenya. Division of Nursing.

[19] Ministry of Health Kenya (2005) Reversing the Trends. The Second National Health Sector Strategic Plan 2005-2010 (NHSSP II).

[20] Mullins, J.L. (2007) Management and Organizational Behavior. 8th Edition, Pearson Education Limited, England.

[21] Nursing Council of Kenya (NCK) (2009) News Letter.

[22] Oppenheim, A.M. (2009) Questionnaire Design, Interviewing and Attitude Measurement. New Edition. Printer Publications. New York.

[23] Pontin, D. and Jones, S. (2007) Children’s Nurses and Nurse Prescribing, a Case Study Identifying Issues for Developing Training Programs. Journal of clinical Nursing, 16, 540-548.

[24] Pullon, et al. (2009) Primary Health Care in Newzealand: The Impact of Organizational Factors on Teamwork. British Journal of General Practice, 59, 191-197. http://dx.doi.org/10.3399/bjgp09X395003

[25] Quinn, M. and Hughes, J. (2007) Quinn’s Principles and Practice of Nurse Education. 5th Edition, Nelson Thornes.

[26] Training and Development Agency for Schools (2007) 151 Buckingham Palace Road, London, SW1W 9SZ TDA switchboard: t 08704960 123Publications: t 08456060323 e publications@tda.gov.ukwww.tda.gov.uk@ TDA 2007.

[27] Winslade, E., et al. (2007) Integrating Performance Assessment, Maintenance of Competence and CPD of Community Pharmacists. The American Journal of Pharmaceutical Education, 71, 15. http://dx.doi.org/10.5688/aj710115

[28] World Health Organization (2010) World Health Assembly 59.27 Strengthening Nursing and Midwifery.

[29] World Health Organization (2008) Ouagadougou Declaration on Primary Healthcare and Health Systems in Africa: Achieving Better Health for Africa in the New Millennium. www.afro.who.int/phc_hs_2008/documents/En/Ougagougou\%20declaration\%20version\%20Eng.pdf 


\section{List of Abbreviations}

AMREF-African research foundation;

CPD-continuing professional development;

CME-continuing medical education;

IEC-information, education and communication;

KDHS-Kenya demographic health surveys;

MDG-millennium development goals;

$\mathrm{MOH}$-ministry of health;

MPA - maternal and perinatal audit;

NCK-Nursing council of Kenya;

WHO-world health organization. 